\title{
Live birth following laparoscopic fertility-sparing surgery for papillary thyroid carcinoma arising from mature ovarian cystic teratoma: A case report
}

\author{
NAOYUKI IWAHASHI ${ }^{1,2}$, YOKO DEGUCHI ${ }^{1,2}$, YUKO HORIUCHI ${ }^{1,2}$, TOMOKO NOGUCHI $^{2}$, \\ TAMAKI YAHATA $^{2}$, NAMI OTA ${ }^{2}$, KAZUHIKO INO ${ }^{2}$ and KENICHI FURUKAWA ${ }^{1}$ \\ ${ }^{1}$ Department of Obstetrics and Gynecology, Hashimoto Municipal Hospital, Hashimoto, Wakayama 648-0005; \\ ${ }^{2}$ Department of Obstetrics and Gynecology, Wakayama Medical University, Wakayama, Wakayama 641-0012, Japan
}

Received August 29, 2018; Accepted October 5, 2018

DOI: $10.3892 / \mathrm{mco} .2018 .1743$

\begin{abstract}
Papillary thyroid carcinoma arising from ovarian mature cystic teratoma is clinically rare. We herein present a case of live birth following two laparoscopic surgeries for papillary thyroid carcinoma arising in a mature ovarian cystic teratoma. A 30-year-old female patient, gravida 1 para 1, was treated by laparoscopic bilateral ovarian cystectomy for suspicion of bilateral mature cystic teratoma. The diagnosis of papillary thyroid carcinoma arising from right ovarian mature cystic teratoma was established based on postoperative pathological examination of the tumor. Such rare neoplasms may be difficult to diagnose preoperatively based on radiological examinations alone. The patient underwent laparoscopic fertility-preserving unilateral (right) salpingo-oophorectomy. Following an extensive discussion with the patient and her family, appropriate informed consent was obtained for the treatment option and the patient and her family chose to preserve her fertility. She could have a baby following the treatment and no evidence of disease for 6 years. Gynecologists should be aware of the possibility of such rare cases, and the available surgical interventions should be fully discussed with patients who wish to preserve their fertility. Laparoscopic fertility-sparing surgery may be a feasible option when encountering such a rare condition.
\end{abstract}

\section{Introduction}

Mature cystic teratoma is a common neoplasm accounting for $10-20 \%$ of all ovarian tumors, and most of ovarian mature cystic teratomas occur in women of reproductive

Correspondence to: Dr Naoyuki Iwahashi, Department of Obstetrics and Gynecology, Wakayama Medical University, 811-1 Kimiidera, Wakayama, Wakayama 641-0012, Japan

E-mail: naoyuki@wakayama-med.ac.jp

Key words: papillary thyroid carcinoma, mature cystic teratoma, laparoscopic surgery, reproductive age, fertility age (1). Although mature cystic teratoma is benign, malignant transformation occurs in $\sim 1-2 \%$ of all cases, often in postmenopausal women with large tumors (2). The lack of characteristic symptoms and specific imaging findings may lead to preoperative misdiagnosis. Although preoperative diagnosis of mature cystic teratoma of the ovary is usually achieved with the use of sonography, computed tomography $(\mathrm{CT})$ and magnetic resonance imaging (MRI), due to the detection of teeth, bones and cartilage (3), malignant transformation of mature cystic teratoma is usually detected incidentally postoperatively based on detailed pathological examination (4). The optimal treatment for malignant transformation of mature cystic teratoma is uncertain due to the rarity of the disease (5).

The most common malignant tumor in mature cystic teratomas is squamous cell carcinoma, whereas papillary thyroid carcinoma arising from mature cystic teratoma is extremely rare (6), with only 11 such cases documented in the English literature to date (5,7-16). In the majority of the cases, malignant transformation is diagnosed on postoperative pathological examination. Only 5 cases of papillary thyroid carcinoma arising from mature cystic teratoma have been reported in younger patients who wished to preserve their fertility (5,7-10), and the optimal clinical management strategy is not always clear due to our limited experience with such cases.

We herein report a rare case of live birth following two laparoscopic surgeries for papillary thyroid carcinoma arising from mature ovarian cystic teratoma. The patient was of reproductive age and the effects of surgical options on fertility were fully discussed when considering treatment.

\section{Case report}

A 30-year-old female patient, gravida 1 para 1, who had her first transvaginal delivery 1 month earlier, was referred to the Department of Obstetrics and Gynecology (Hashimoto Municipal Hospital, Wakayama, Japan) in April 2011 for a routine gynecological examination. Transvaginal ultrasound examination revealed bilateral cystic ovarian tumors, sized $2.7 \mathrm{~cm}$ on the right and $3.2 \mathrm{~cm}$ on the left side, which 
contained high-echogenicity regions. The tumor size grew to $4.2 \mathrm{~cm}$ on the right and $6.1 \mathrm{~cm}$ on the left side over the next 3 months. A CT scan revealed bilateral ovarian tumors with a partial solid component and calcification, without pelvic lymph node enlargement (Fig. 1). MRI could not be performed, as the patient had a cardiac pacemaker. Based on the imaging findings, the suspected diagnosis was bilateral ovarian teratoma. The results of the laboratory tests revealed normal levels of carbohydrate antigen (CA) 125 and carcinoembryonic antigen, and an elevated CA19-9 level to $70.0 \mathrm{U} / \mathrm{ml}$ (normal level $\sim 37.0 \mathrm{U} / \mathrm{ml}$ ). The serum biochemical examination and coagulation profile were within normal limits. The serum level of thyroid-stimulating hormone was also within normal limits. Laparoscopic bilateral cystectomy was performed. An intraoperative examination revealed bilateral ovarian cystic tumors, which were mobile and had a smooth surface, both of which were enucleated; there were no adhesions or invasion of other organs (Fig. 2A and B). The tumors were placed in a plastic bag and ruptured, and the contents of the bag were aspirated and removed through the umbilical trocar site. No free fluid was detected in the peritoneal cavity. No frozen sections were prepared for pathological examination.

Macroscopically, the bilateral cystic ovarian tumors exhibited the typical structure of dermoid cysts containing hair and fat (Fig. 2C). Microscopically, the left tumor was a mature cystic teratoma containing hair follicles, adipose, bone, cartilaginous and brain tissue, whereas the right tumor was a mature cystic teratoma with a thyroid gland component displaying papillary structure (Fig. 3). The tumor cells had oval or round overlapping nuclei with a ground glass appearance. Immunohistochemical examination revealed positive staining of the tumor cells for cytokeratin (CK) 19, and Hector Battifora mesothelial epitope-1 (HBME-1). The diagnosis of papillary thyroid carcinoma arising from ovarian mature cystic teratoma was confirmed based on the pathological findings.

In order to exclude distant metastasis, positron emission tomography (PET)-CT was performed. PET-CT revealed normal thyroid, lung, liver, pancreas, spleen, kidneys and retroperitoneal space. No enlarged lymph nodes were identified in the intra-abdominal and retroperitoneal space. The surgical options were fully discussed with the patient, in order to decide whether to perform right unilateral salpingo-oophorectomy or complete surgery for ovarian cancer (total abdominal hysterectomy, bilateral salpingo-oophorectomy, omentectomy, pelvic lymphadenectomy and para-aortic lymphadenectomy), and she opted for unilateral salpingo-oophorectomy to preserve her fertility. One month after the first laparoscopic surgery, laparoscopic right unilateral salpingo-oophorectomy was performed. No free fluid was detected in the peritoneal cavity, and the peritoneal lavage cytology was negative. The right ovary was normal in size, without adhesions or obvious invasion of other organ (Fig. 2D). On histopathological examination, the right ovary was free from cancer infiltration. Adjuvant chemotherapy was not administered and the patient gave birth following natural conception without any problems 2 years following surgery. There was no evidence of local recurrence or systemic disease in September 2017.

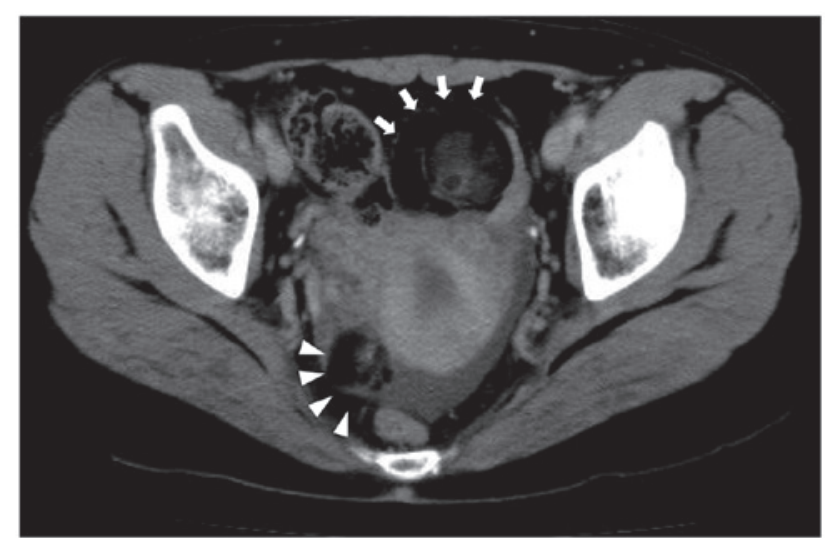

Figure 1. Computed tomography findings of papillary thyroid carcinoma arising in a mature cystic ovarian teratoma (arrowheads, right ovary; arrows, left ovary).

\section{Discussion}

The majority of mature cystic teratomas are composed of well-differentiated derivatives of the three germ layers, namely the ectoderm, the mesoderm and the endoderm, while ectodermal elements usually predominate, and it has been reported that $\sim 15 \%$ of the tumors contain thyroid tissue (6). The complications of mature cystic teratoma include torsion (16\%), malignant degeneration (2\%), rupture (1-2\%) and infection (1\%) (17), whereas uncomplicated teratomas are found incidentally on routine gynecological examination in asymptomatic patients. The main concern with treating mature cystic teratoma is the possibility of malignant transformation, which can only be correctly diagnosed postoperatively based on detailed pathological examination. Particularly in women of reproductive age, malignant transformation of mature cystic teratoma is even more uncommon. The survival outcome of patients with malignant transformation of mature cystic teratoma depends on the stage at diagnosis.

Papillary thyroid cancer arising from mature cystic teratoma is a rare occurrence, with an estimated incidence of $0.1-0.3 \%$ (18), with 11 cases in total reported in the literature to date (5,7-16), among which only 5 were patients of reproductive age (5,7-10) according to a search of the English literature on PubMed and MEDLINE. In patients of reproductive age, including the case in the present study (5,7-10), the mean age and tumor size were 29.2 years (range, 19-39 years) and $8.62 \mathrm{~cm}$ (range, 4.2-15 cm), respectively. In two cases the patients were asymptomatic and the tumors were discovered incidentally $(5,10)$. To the best of our knowledge, the tumor in the present case was the smallest among all reported asymptomatic cases of reproductive age, indicating that the size of the tumor and the presence of symptoms are not indicative of the diagnosis.

The most appropriate clinical management strategy for papillary thyroid carcinoma arising from mature cystic teratoma is not always clear, as our experience with such cases is limited. Laparoscopic surgery is usually selected when the gynecologist suspects mature cystic teratoma, as it is a minimally invasive surgery, and it may also be possible to treat patients wishing to preserve their fertility by fertility-sparing surgery including unilateral salpingo-oophorectomy or cystectomy without adjuvant chemotherapy. According to previous reports on the 

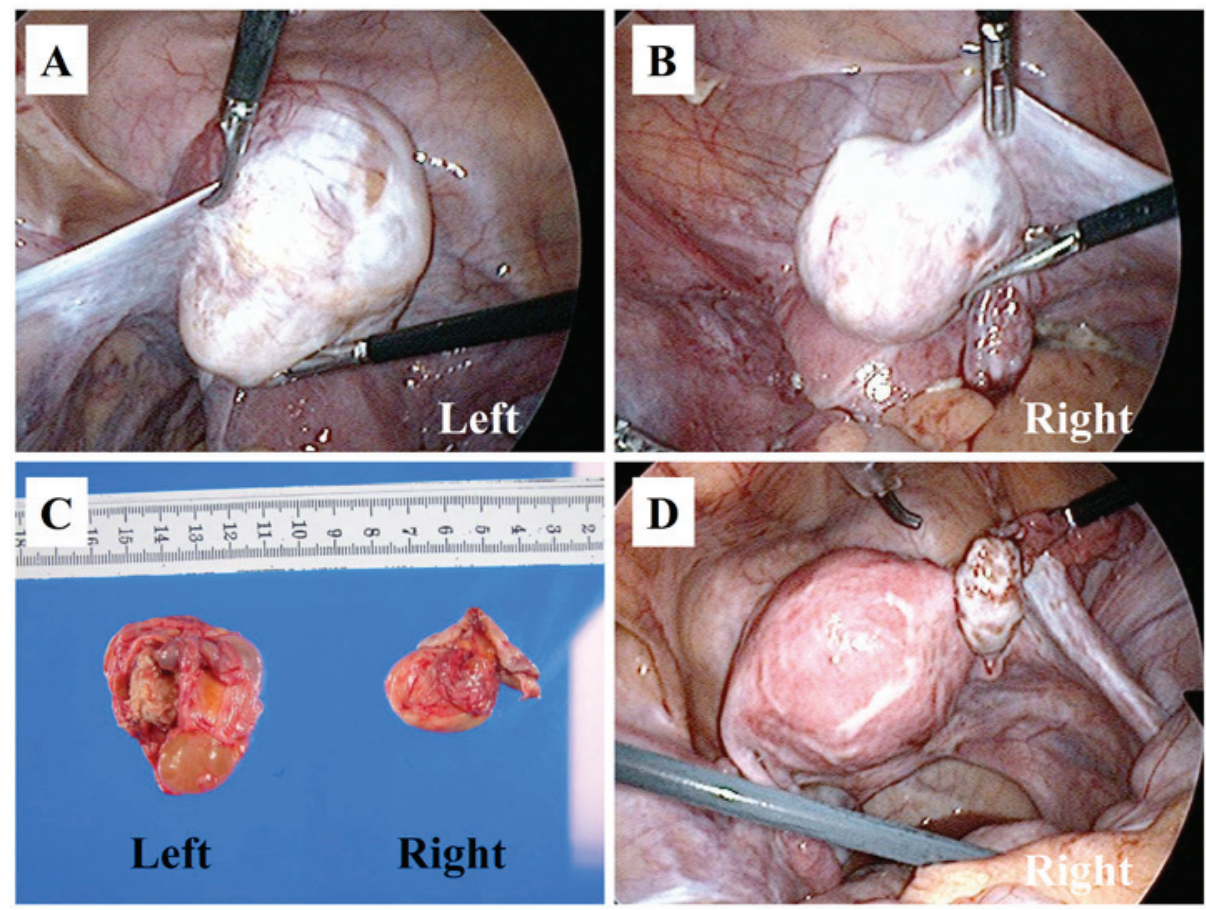

Figure 2. Laparoscopic and macroscopic findings of papillary thyroid carcinoma arising in a mature cystic teratoma. (A) Left ovarian tumor on the first surgery. (B) Right ovarian tumor on the first surgery. (C) Macroscopic appearance of the resected tumors. (D) Right ovary on the second surgery.
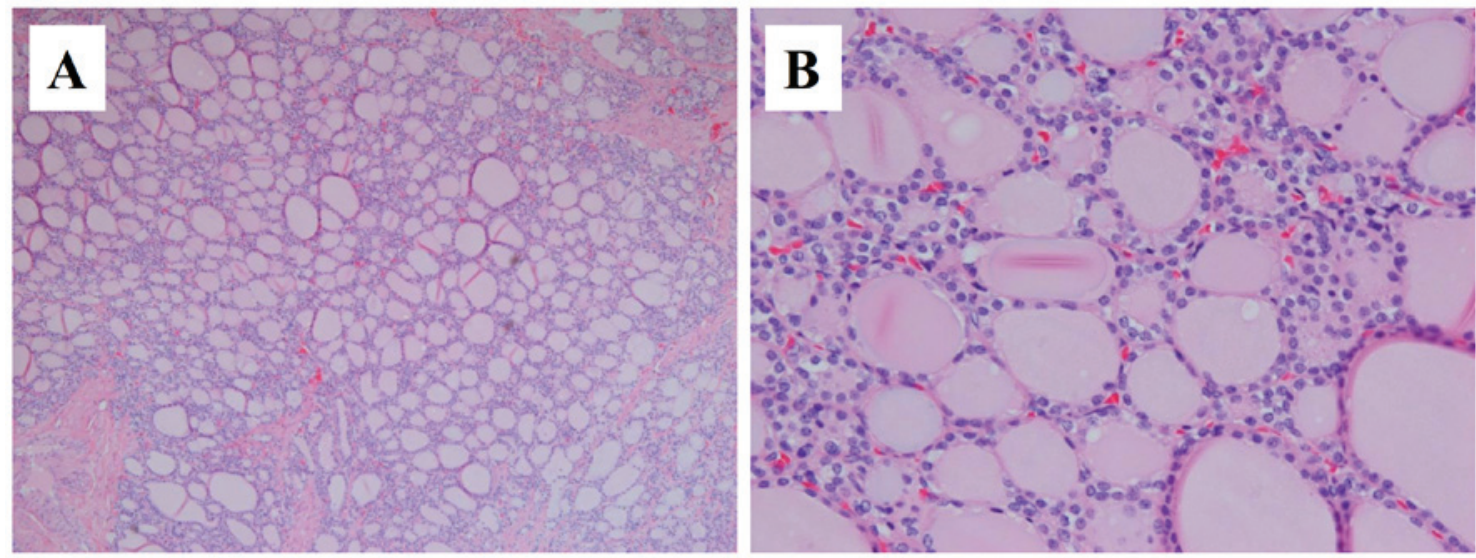

Figure 3. Microscopic findings of papillary thyroid carcinoma arising in a mature cystic teratoma. Hematoxylin and eosin staining; magnification (A) $\mathrm{x} 40$ and (B) $\times 200$.

condition, abdominal cystectomy was performed in 2 cases $(7,8)$, laparoscopic salpingo-oophorectomy in 1 case (5), laparoscopic salpingo-oophorectomy followed by total abdominal hysterectomy with contralateral salpingo-oophorectomy in 1 case (9), and abdominal cystectomy followed by abdominal adnexectomy in 1 case (10). The long-term prognosis of papillary thyroid carcinoma arising from mature cystic teratoma is unclear, with a limited number of reports on follow-up time (6-33 months). The prognosis appears to be favorable, as no metastasis or recurrence have been reported. In the present case, laparoscopic bilateral cystectomy was first performed, followed by unilateral salpingo-oophorectomy. The options were fully discussed with the patient and their family and written informed consent was obtained. The patient was advised that she would be able to become pregnant following treatment, provided there was no evidence of disease for 6 years. To improve the treatment of papillary thyroid carcinoma arising from mature cystic teratoma in the future, further data regarding its natural history, diagnosis and treatment outcome are needed.

In summary, we herein present a rare case of papillary thyroid carcinoma arising from a mature cystic ovarian teratoma, in which two laparoscopic surgeries was performed and the patient was able to successfully carry a pregnancy to term after treatment. Gynecologists should be aware of the possibility of such rare cases, and the available surgical interventions should be fully discussed with patients who wish to preserve their fertility. Laparoscopic fertility-sparing surgery may be a viable treatment option when encountering such rare cases.

\section{Acknowledgements}

Not applicable. 


\section{Funding}

No funding was received.

\section{Availability of data and materials}

The datasets obtained and/or analyzed during the present study are available from the corresponding author on reasonable request.

\section{Authors' contributions}

NI and YD diagnosed, investigated and managed the patient. NI wrote the initial draft of the report. NI, YD and $\mathrm{YH}$ acquired the data in the surgical field. TN, TY and NO acquired the data in the diagnostic imaging and pathological examination. KI and KF supervised the study and critically reviewed the manuscript. All authors have read and approved the final version of the manuscript.

\section{Ethics approval and consent to participate}

Written informed consent for surgery was obtained from the patient.

\section{Patient consent for publication}

Written informed consent for publication of the case details and associated images was obtained from the patient.

\section{Competing interests}

The authors declare that they have no competing interests.

\section{References}

1. Lai PF, Hsieh SC, Chien JC, Fang CL, Chan WP and Yu C: Malignant transformation of an ovarian mature cystic teratoma: Computed tomography findings. Arch Gynecol Obstet 271: 355-357, 2005.

2. Wen KC, Hu WM, Twu NF, Chen P and Wang PH: Poor prognosis of intraoperative rupture of mature cystic teratoma with malignant transformation. Taiwan J Obstet Gynecol 45 253-256, 2006.

3. Kido A, Togashi K, Konishi I, Kataoka ML, Koyama T, Ueda H, Fujii S and Konishi J: Dermoid cysts of the ovary with malignant transformation: MR appearance. AJR Am J Roentgenol 172: 445-449, 1999
4. Mori Y, Nishii H, Takabe K, Shinozaki H, Matsumoto N, Suzuki K, Tanabe H, Watanabe A, Ochiai K and Tanaka T: Preoperative diagnosis of malignant transformation arising from mature cystic teratoma of the ovary. Gynecol Oncol 90: 338-341, 2003.

5. Lataifeh I, Abdel-Hadi M, Morcos B, Sughayer M and Barahmeh S: Papillary thyroid carcinoma arising from mature cystic teratoma of the ovary. J Obstet Gynaecol 30: 884-886, 2010.

6. Peterson WF: Malignant degeneration of benign cystic teratomas of the overy; a collective review of the literature. Obstet Gynecol Surv 12: 793-830, 1957.

7. Dane C, Ekmez M, Karaca A, Ak A and Dane B: Follicular variant of papillary thyroid carcinoma arising from a dermoid cyst: A rare malignancy in young women and review of the literature. Taiwan J Obstet Gynecol 51: 421-425, 2012.

8. Soto Moreno A, Venegas EM, Rodriguez JR, Sánchez F, Robles MJ, Martinez MA, Gonzalez D, Navarro E and Astorga R: Thyroid carcinoma on an ovarian teratoma: A case report and review of the literature. Gynecol Endocrinol 16: 207-211, 2002.

9. Lee JM, Kim JW, Song JY, Lee JK, Lee NW, Kim SH and Lee KW: Adenocarcinoma arising in mature cystic teratoma: A case report. J Gynecol Oncol 19: 199-201, 2008.

10. Cymbaluk-Ploska A, Chudecka-Głaz A, Chosia M, Ashuryk O and Menkiszak J: Conservative treatment of a young patient with thyroid carcinoma in adult ovarian teratoma-case report. Gynecol Endocrinol 30: 187-191, 2014.

11. Chadha S and Schaberg A: Malignant transformation in benign cystic teratomas: Dermoids of the ovary. Eur J Obstet Gynecol Reprod Biol 29: 329-338, 1988.

12. Doldi N, Taccagni GL, Bassan M, Frigerio L, Mangili G, Jansen AM and Ferrari A: Hashimoto's disease in a papillary carcinoma of the thyroid originating in a teratoma of the ovary (malignant struma ovarii). Gynecol Endocrinol 12: 41-42, 1998.

13. Bal A, Mohan H, Singh SB and Sehgal A: Malignant transformation in mature cystic teratoma of the ovary: Report of five cases and review of the literature. Arch Gynecol Obstet 275: 179-182, 2007.

14. Ryder M, Nikiforov YE and Fagin JA: Follicular variant papillary thyroid carcinoma arising within an ovarian teratoma. Thyroid 17: 179-180, 2007.

15. Quadri AM, Ganesan R, Hock YL, Karim SN and Hirschowitz L: Malignant transformation in mature cystic teratoma of the ovary: Three cases mimicking primary ovarian epithelial tumors. Int J Surg Pathol 19: 718-723, 2011.

16. Tanaka H, Sakakura Y, Kobayashi T, Yoshida K, Asakura T and Taniguchi H: A case of thyroid-type papillary carcinoma derived from ovarian mature cystic teratoma, resected by laparoscopic surgery. Asian J Endosc Surg 4: 86-89, 2011.

17. Lipson SA and Hricak H: MR imaging of the female pelvis. Radiol Clin North Am 34: 1157-1182, 1996.

18. Rim SY, Kim SM and Choi HS: Malignant transformation of ovarian mature cystic teratoma. Int J Gynecol Cancer 16: 140-144, 2006.

This work is licensed under a Creative Commons Attribution-NonCommercial-NoDerivatives 4.0 International (CC BY-NC-ND 4.0) License. 